\title{
Atrial fibrillation: the last challenge in interventional electrophysiology
}

The number of antiarrhythmic drugs currently used to treat atrial fibrillation reflects the inadequacy of the available drugs - no agent approaches the ideal in terms of efficacy, tolerability, and safety. Whether the goal of treatment is the maintenance of sinus rhythm or control of the ventricular rate, in many it cannot be achieved with drugs alone. This group is proportionately small but numerically important - every cardiologist sees such patientsand it is not surprising that increasing attention is being paid to possible alternatives to drug treatment.

\section{Control of the ventricular rate: atrioventricular nodal ablation and modification \\ PERMANENT ATRIAL FIBRILLATION}

For patients with irreversible atrial fibrillation and a rapid ventricular response that is refractory to drug treatment, catheter ablation of the atrioventricular node is now an established approach. Procedural success rates are high and complications rare in experienced hands. ${ }^{1}$ With appropriate selection of patients, an improved quality of life can be achieved. Modest increases in left ventricular ejection fraction and exercise tolerance have been reported..$^{2-4}$

The disadvantage of atrioventricular node ablation is that it creates permanent pacemaker dependence: it would be better to slow the ventricular response by modulating atrioventricular conduction, rather than interrupt it altogether. ${ }^{5}$ Attempts to achieve this by reducing the energy delivered using the standard (anterior) approach to the atrioventricular node have been disappointing, yielding either complete heart block or unchanged atrioventricular conduction during atrial fibrillation. ${ }^{6}$ More recently, modification of atrioventricular conduction has been reported with ablation at a posteroseptal site, equivalent to the "slow pathway", in patients with dual atrioventricular physiology. ${ }^{78}$ The rationale for this approach is that the slowly conducting inputs to the atrioventricular node generally have the shortest refractory period and that this property, rather than conduction velocity, determines the ventricular rate during atrial fibrillation. This model may be too simple: the function of the node and its inputs remains unclear, and dual nodal physiology is certainly not universal. Ablation sites that achieve a sustained reduction in ventricular rate lie close to the compact node itself, so that efficacy carries a significant risk of complete heart block. The role of atrioventricular nodal modification will undoubtedly become clearer as many centres attempt this technique. For the time being, it should be reserved for patients in whom pacemaker dependence is an acceptable outcome.

\section{PAROXYSMAL ATRIAL FIBRILLATION}

The conceptual simplicity of atrioventricular nodal ablation for chronic atrial fibrillation has led some to regard it as a panacea for "difficult" patients, and the procedure is increasingly performed in those with symptomatic paroxysmal atrial fibrillation that is resistant to drug treatment.
However, management of such patients is far from straightforward. Dual chamber pacing is required because atrioventricular dissociation during sinus rhythm is both poorly tolerated and arrhythmogenic. The device implanted, however, should also be able to switch mode to some form of physiological ventricular pacing (VVIR) during episodes of atrial fibrillation to avoid tracking the arrhythmia at a fast ventricular rate. Such state-of-the-art devices require considerable fine tuning to avoid abrupt fluctuations in heart rate. The symptomatic results of atrioventricular nodal ablation for paroxysmal atrial fibrillation can be spectacularly successful but are often disappointing, even with these precautions, perhaps because patients frequently remain acutely aware of changes in atrial rhythm. Furthermore, atrial fibrillation is not an entirely benign arrhythmia. Apart from its thromboembolic complications, it has adverse haemodynamic effects even in patients with a controlled ventricular rate. ${ }^{9}$ Atrioventricular nodal ablation is inappropriate for paroxysmal atrial fibrillation unless symptoms are clearly related to a rapid ventricular rate and all other therapeutic options have been considered.

Restoring and maintaining sinus rhythm with devices The restoration and preservation of sinus rhythm, where feasible, remains the preferred goal of treatment in patients with atrial fibrillation. Unfortunately, currently available antiarrhythmic drugs are all of limited efficacy and none is entirely safe. The newer class III agents (for example, $d$-sotalol, dofetilide, azimilide, ibutilide) seem to have substantial potency in the atrium, but the high hopes held out for them must be qualified by uncertainty over their potential for causing torsade de pointes. ${ }^{10}$

\section{ATRIAL DEFIBRILLATORS}

An implantable atrial defibrillator might be of considerable value to patients with sustained symptomatic episodes of paroxysmal atrial fibrillation. Early experiments with low energy shocks delivered within the right atrium were ineffective in restoring sinus rhythm, ${ }^{11}$ and the idea was not initially pursued. More recent studies in sheep, however, indicate that low thresholds for transvenous atrial defibrillation can be achieved with electrode configurations that embrace both atria, especially from the high right atrium and coronary sinus. ${ }^{12}$ A further reduction in threshold can be achieved using a biphasic shock waveform. ${ }^{13}$ An initial experience using crude electrodes in patients with paroxysmal atrial fibrillation undergoing electrophysiological study demonstrated a defibrillation threshold around $2 \mathrm{~J}-100$ times lower than that of conventional cardioversion..$^{14}$ It remains to be seen whether modifications to the number, design, and precise location of electrodes, and to the shock waveform can further reduce the defibrillation threshold. It is also not known whether a series of smaller shocks can achieve the same cumulative likelihood of success as a single shock above the "threshold". 


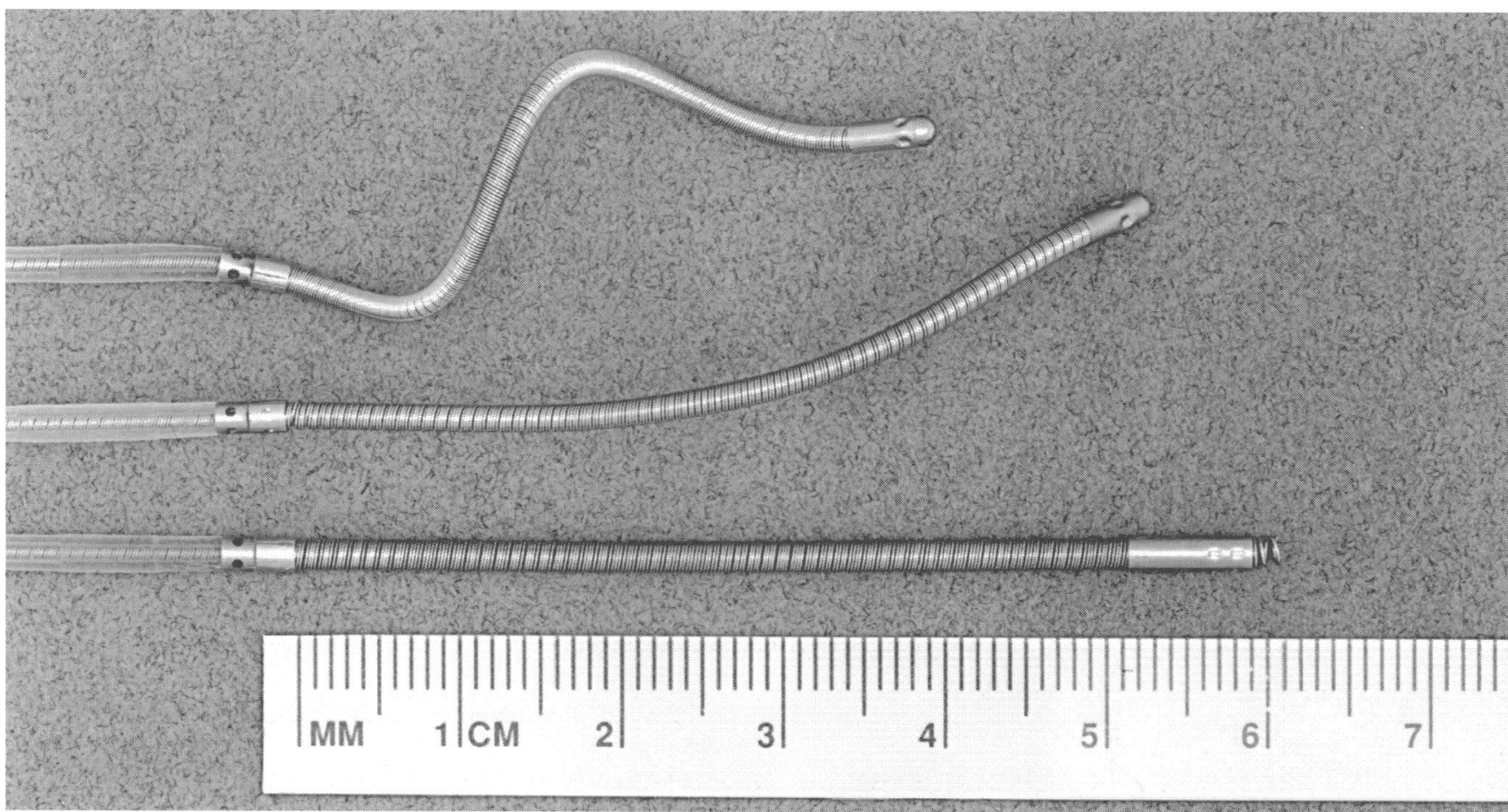

Leads developed for use with an implantable atrial defibrillator. Each has a $5 \mathrm{~cm}$ coil electrode. The coronary sinus lead (middle) is stabilised against the vessel wall by the helical form that it assumes (top) as its stylet is withdrawn. The right atrial lead (bottom) has a conventional active fixation tip.

The technical feasibility of an atrial defibrillator has been demonstrated with a prototype implanted in sheep..$^{15}$ Specially designed leads (figure) are used to obtain stable positions in the right atrium and coronary sinus. The shocks delivered can be smaller and the charge times longer (because atrial fibrillation is not acutely life-threatening) than with ventricular defibrillators. Therefore battery and capacitor size have been reduced so that the device is not much larger than a complex pacemaker, and is suitable for routine prepectoral implantation. The first devices are expected to be implanted in patients some time in 1995. Whether defibrillators can become a standard treatment for patients with "difficult" atrial fibrillation will depend on the outcome of this initial experience in terms of safety and tolerability.

Mechanical complications of shocks delivered by an atrial defibrillator are unlikely. None has been seen with the higher energies used by implantable cardioverterdefibrillator systems, including those with coronary sinus leads. Of more concern is the risk that atrial shocks may initiate ventricular arrhythmias. Even with QRS synchronisation, there is a possibility that a shock delivered after a short $R R$ interval may fall within the relative refractory period of part of the ventricle. Animal studies suggest that the risk of ventricular proarrhythmia appears to be confined to shocks that follow $R R$ intervals of less than $300 \mathrm{~ms}^{16}$ Healthy animals, however, may differ from patients with heart disease. Clinical experience with low energy shocks delivered between right atrium and coronary sinus during atrial fibrillation totalled some 1200 shocks in almost 100 patients by early $1994,{ }^{17}$ and this experience has probably trebled since then. To date, no pro-arrhythmia has been seen with synchronised shocks, though significant pauses can occur in the few seconds that follow shocks, and backup pacing will be a necessary feature of the implanted device. While this safety experience is encouraging, it is as yet insufficient, and the first devices will have to be physician activated. The arrhythmogenic risk must be demonstrated to be at least as low as that from antiarrhythmic drugs-say $1 \%$ per year. The cumulative risk of shocks will depend on their frequency, which is likely to vary considerably between patients, and the effect of structural heart disease on the risk is unknown. The causal link between shocks and any ventricular arrhythmias that occur would be easy to establish from electrograms stored by the device itself. While the inherent safety of low energy defibrillation can be tested in acute studies, certain technical aspects of the system can only be tested in patients. These include the longterm stability of leads and their defibrillation thresholds. Most importantly, the detection algorithms used by the device will be new in many respects, and specificity will be all important to minimise the risk of inappropriate shocks.

Data on the tolerability of low energy intracardiac shocks are limited because few studies of atrial defibrillation have been conducted in conscious patients. Though the atrial defibrillation thresholds achieved so far are remarkably low they still seem to lie slightly above the threshold of pain for most patients. ${ }^{14}$ The effects of posture, lead position, and shock waveform on discomfort are unknown. It is clear from experience with implantable defibrillators that the perception of ventricular shocks varies greatly between patients, and with the circumstance in which shocks are given. While atrial shocks of around $1 \mathrm{~J}$ may be intolerable during an experimental protocol, they may be acceptable to a patient who is familiar with them, able to control their timing, and who associates them with the reliable termination of a highly symptomatic arrhythmia. Fully automatic function of the device, though technically possible, may not be appropriate unless shocks can be made virtually imperceptible.

\section{PACEMAKERS TO TREAT ATRIAL FIBRILLATION}

Another prospect is the implantation of pacemakers to treat atrial fibrillation. It is clear from many retrospective analyses and a recent controlled trial that pacing the atrium in patients with sinus node disease results in a considerably lower incidence of atrial fibrillation than does pacing the ventricle.$^{18}$ It is also common experience that 
episodes of atrial fibrillation sometimes occur less frequently when patients with the sick sinus syndrome are paced. In a small series of patients with paroxysmal atrial fibrillation associated with bradycardia, dual chamber pacing reduced the frequency of episodes, ${ }^{19}$ and randomised trials of atrial pacing in less selected patients are under way. Overdrive pacing at a rate determined by the frequency of atrial premature beats appears promising, but long-term efficacy has not been demonstrated..$^{20}$ "Resynchronisation" by pacing both the right atrium and the coronary sinus can be beneficial in patients with a form of atrial flutter dependent on interatrial conduction block. ${ }^{21}$ Studies in patients with atrial fibrillation are under way, but obtaining satisfactory and stable coronary sinus pacing is not always straightforward. Finally, local capture of atrial fibrillation in dogs has been described by one centre. ${ }^{22}$ If this can be reproduced in patients, it is possible that sophisticated pacing devices might one day be able to simultaneously entrain sufficient atrial myocardium to extinguish atrial fibrillation. This objective remains distant, but if it is achieved it will be a triumph of technology over human electrophysiology.

\section{Modification of the atrial substrate}

The ability to reproduce the new atrial fibrillation operations ${ }^{23}$ by catheter ablation is an enticing prospect. In particular, a non-thoracotomy "maze" procedure would be an attractive treatment for many patients: the current operation restores both sinus rhythm and a degree of atrial transport in most patients, but it is a major surgical undertaking with a significant morbidity. Established radiofrequency techniques produce point lesions, typically $5 \mathrm{~mm}$ in diameter, rather than the linear lesions necessary for the abolition of atrial fibrillation. The development of catheter based techniques to deliver several such lesions within both atria (probably using a transseptal approach for the left) with sufficient precision presents a considerable technical challenge. Two approaches are being taken. New catheters have been developed with long flexible tips that produce linear, longitudinal lesions. ${ }^{24}$ However, is not yet clear that continuous surface contact can be consistently achieved, nor how such catheters can be made sufficiently steerable to locate lesions correctly within the atria. The alternative approach is to produce a linear lesion by dragging a conventional ablation catheter along a preset path on the endocardium. This method has recently been described in a few patients, using conventional ablation catheters guided by a series of shaped sheaths to produce multiple linear lesions approximating those of the maze operation. ${ }^{25}$ At present, the procedure is lengthy with long screening times-indeed, it is often performed over two or more days - and may result in atrial flutter circuits that themselves require ablation. Nevertheless, it has demonstrated the feasibility of using radiofrequency lesions to abolish atrial fibrillation. The next logical step would be to develop an entirely new procedure based on steerable catheters, rather than attempting to copy a surgical technique.

The era of interventional therapy for atrial fibrillation is approaching, but gradually. The rate of implantation of devices to prevent and treat atrial fibrillation is unlikely to mirror the explosive growth seen with ventricular defibril- lators, in which the possibility of saving a life makes a bulky, imperfect, and even painful device acceptable. However, if the safety and tolerability of atrial defibrillators or the efficacy of atrial pacing can ever be demonstrated, such implanted devices may considerably improve the quality of life for many patients. The number of such devices actually used will then depend partly on the willingness of health care purchasers to pay for this improvement and partly on advances made in catheter based techniques to modify the atrial substrate.

FRANCIS D MURGATROYD

Department of Cardiological Sciences,

A JOHN CAMM

St George's Hospital Medical School,

London SW17 ORE

1 Hindricks G. The Multicentre European Radiofrequency Survey (MERFS): complications of radiofrequency catheter ablation of arrhythmias. The Multicentre European Radiofrequency Survey (MERFS) investigators of the Working Group on Arrhythmias of the European Society of Cardiology. Eur Heart f 1993;14:1644-53.

2 Kay GN, Bubien RS, Epstein AE, Plumb VJ. Effect of catheter ablation of the atrioventricular junction on quality of life and exercise tolerance in the atrioventricular junction on quality of life and exercise

3 Brignole M, Gianfranchi L, Menozzi C, et al. Influence of atrioventricular junction radiofrequency ablation in patients with chronic atrial fibrillation and flutter on quality of life and cardiac performance. Am 7 Cardio 1994;74:242-6.

4 Twidale N, Sutton K, Bartlett L, et al. Effects on cardiac performance of atrioventricular node catheter ablation using radiofrequency current for drug-refractory atrial arrhythmias. PACE 1993;16:1275-84.

5 McComb JM, McGovern B, Garan H, Ruskin JN. Management of refractory supraventricular tachyarrhythmias using low-energy transcatheter shocks. Am ₹ Cardiol 1986;58:959-63.

6 Duckeck W, Engelstein ED, Kuck KH. Radiofrequency current therapy in atrial tachyarrhythmias: modulation versus ablation of atrioventricular nodal conduction. $P A C E$ 1993;16:629-36.

7 Williamson BD, Man KC, Daoud E, Niebauer M, Strickberger SA, Morady F. Radiofrequency catheter modification of atrioventricular
conduction to control the ventricular rate during atrial fibrillation. conduction to control the vent
$N$ Engl $f$ Med 1994;331:910-7.

8 Feld GK, Fleck RP, Fujimura O, Prothro DL, Bahnson TD, Ibarra M. Control of rapid ventricular response by radiofrequency catheter modifiControl of rapid ventricular response by radiofrequency catheter modifi-
cation of the atrioventricular node in patients with medically refractory cation of the atrioventricular node in patients with

9 Gosselink ATM, Crijns HJ, Van Den Berg MP, et al. Functional capacity before and after cardioversion of atrial fibrillation: a controlled study. Br Heart $\mathcal{F}$ 1994;72:161-6.

10 Katritsis D, Camm AJ. New class III antiarrhythmic drugs. Eur Heart $\mathcal{f}$ 1993;14(H):93-9.

11 Nathan AW, Bexton RS, Spurrell RAJ, Camm AJ. Internal transvenous low energy cardioversion for the treatment of cardiac arrhythmias. Br Heart $\mathcal{F}$ 1984;52:377-84.

12 Cooper RA, Alferness CA, Smith WM, Ideker RE. Internal cardioversion of atrial fibrillation in sheep. Circulation 1993;87:1673-86.

13 Keane D, Boyd E, Anderson D, et al. Comparison of biphasic and monophasic waveforms in epicardial atrial defibrillation. $\mathcal{F} \mathrm{Am}$ Coll Cardiol 1994;24:171-6.

14 Murgatroyd FD, Slade AKB, Sopher SM, Rowland E, Ward DE, Camm AJ. Efficacy and tolerability of transvenous low energy cardioversion of AJ. Efficacy and tolerability of transvenous low energy cardioversion of
paroxysmal atrial fibrillation in man. $\mathcal{F} \mathrm{Am}$ Coll Cardiol 1995;25: paroxysmal

15 Ayers GM, Griffin JC, Ilina MB, et al. An implantable atrial defibrillator: initial experience with a novel device [abstr]. PACE 1994;17:769.

16 Ayers GM, Alferness CA, Ilina M, et al. Ventricular proarrhythmic effects of ventricular cycle length and shock strength in a sheep model of transvenous atrial defibrillation. Circulation 1994;89:413-22.

17 Murgatroyd FD, Johnson EE, Cooper RA, et al. Safety of low energy transvenous atrial defibrillation-world experience [abstr]. Circulation 1994;90:14.

18 Andersen HR, Thuesen L, Bagger JP, Vesterlund T, Thomsen PEB. Prospective randomized trial of atrial versus ventricular pacing in sicksinus syndrome. Lancet 1994;344:1523-8.

19 Attuel P, Pellerin D, Mugica J, Coumel P. DDD pacing: an effective treatment modality for recurrent atrial arrhythmias. $P A C E$ 1988;11:1647-54.

20 Murgatroyd FD, Nitzsche $R$, Slade $A K B$, et al. A new pacing algorithm for overdrive suppression of atrial fibrillation. PACE 1994;17:1966-73.

21 Daubert C, Mabo P, Berder V, Gras D, Leclercq C. Atrial tachyarrhythmias associated with high degree interatrial conduction block: prevention by permanent atrial resynchronization. European fournal of Cardiac tion by permanent atrial resynchronization.
Pacing and Electrophysiology 1994;4:35-44.

22 Kirchhof C, Chorro F, Scheffer GJ, et al. Regional entrainment of atrial fibrillation studied by high-resolution mapping in open-chest dogs. fibrillation studied by high-

$23 \mathrm{McComb}$ JM. Surgery for atrial fibrillation. Br Heart 7 1994;71:501-3.

24 Avitall B, Hare J, Mughal K, Silverstein E, Krum DP. A catheter system to blate atrial fibrillation in a sterile pericarditis dog model [abstr]. $P A C E$ 1994;17:774.

25 Swartz JF, Pellersels G, Silvers J, Patten L, Cervantez D. A catheter-based curative approach to atrial fibrillation in humans [abstr]. Circulation 1994;90:I-335. 